\title{
A New Stochastic Geometry Model of Coexistence of Wireless Body Sensor Networks
}

\author{
Ming Yang ${ }^{1}$, Ruixia Liu ${ }^{1,2^{*}}$, Yinglong Wang ${ }^{1,2}$, Minglei Shu ${ }^{1}$ and Yuanyuan Yang ${ }^{1}$ \\ ${ }^{1}$ Shandong Computer Science Center(National Supercomputer Center in Jinan), Shandong Provincial Key Laboratory of \\ Computer Networks, Ji'nan 250014, China \\ ${ }^{2}$ College of Information Science and Engineering, Shandong University of Science and Technology, Qingdao 266590, \\ China \\ Corresponding Email: liurx@sdas.org
}

\begin{abstract}
Stochastic geometry, in particular Poission point process theory, has been widely used in the last decade to provide models and methods to analyze wireless networks. It is a branch of mathematics which deals with the study of random point processes. There are various models for point processes, typically based on but going beyond the classic homogeneous Poisson point process. Poisson point process cannot be used to model the spatial distribution of the simultaneously active transmitters. A novel framework has been presented for modeling the intensity of simultaneous active transmitters of a random carrier sense multiple access wireless sensor network. This thinning rule uses a second-neighbors distance-dependent method, which controls too many nodes deleted of points close together.
\end{abstract}

\section{Introduction}

WBSNs enable wireless communications between several miniaturized body sensors and a single coordinator worn on the human body. WBSNs offer many promising new applications in the area of remote health monitoring systems to measure specified physiological data and also provide location-based information. Since a WBSNs provide remote and continuous medical monitoring for patients without constraining their movements, it plays a crucial role in next generation healthcare applications [1-2].

WBSNs for health monitoring systems are required to meet stringent performance demands regarding the tradeoff between reliability, latency, and power efficiency. WBSNs feature limited range and bandwidth and they are prone to interference. These modes incur issues regarding the coexistence of multiple WBANs. There is often a group of BSNs together to happen in hospitals for patients and staff or for the elderly at nursing homes [3]. In many cases, several WBSNs coexist in a small area, resulting in very strong inter-BAN interference, which seriously disturbs intra-BAN communications [4].

In this paper, we use stochastic geometry to analyze the coexistence of IEEE802.15.4-based WBSNs Stochastic geometry is an established branch of mathematics that studies uncertainty in geometric structures. The density of effective points in model is a sufficient parameter to describe the network performance. However, The HCPP model still suffers from the underestimation problem. So we proposed a PCMP model to mitigate this problem.

We propose a new thinning method of CSMA network to mitigate the node intensity underestimation problem of HCPP model. We put forward a modified Poisson cluster and marked process (PCMP) to adapt to this network coexistence The PCMP model is generalized for CSMA protocol's network.

Define:

Contention domain: node $\mathrm{y}$ is in the contention domain of node $\mathrm{x}$ if the power received by $\mathrm{x}$ from $\mathrm{y}$ is above some detection threshold.

Neighbors: the neighbors of a node are the nodes in its contention domain.

Second Neighbors: the neighbors of a origin node's neighbors node.

The rest of the paper is organized as follows: Section 2 introduces the related work. Section 3 proposes and describes the system model in detail. In Section 4, thinning theory and stochastic geometry are used to investigate the CSMA-based wireless sensor networks coexistence. Section 5 illustrates and analyzes numerical results. We conclude our work in Section 6.

\section{Related work}


Since the Poisson Point Process (PPP) is highly tractable, it is frequently used to model a variety of networks, such as celluar networks, mobile ad hoc networks, cognitive radio networks and wireless sensor networks[5-6]. In [7], the authors assume the star topology network's transmission nodes to be uniformly distributed according to a PPP. this paper stands in the precise consideration of CSMA algorithms, considering the collision, the success transmit probability and interference in the same network. The matern hard core are often used to model concurrent transmitters in CSMA networks [8-9]. Hesham EISawy et al. [10] adopt the Poisson cluster process to obtain the minimum required number of channels of the coexisting IEEE 802.15.4 networks, but the underestimation problem is not fully resoved.

\section{System model}

The carrier sense multiple access with collision avoidance (CSMA-CA) is widely employed in wireless networking due to its simplicity and performance efficiency [11].

CSMA create exclusion zones to protect scheduled transmissions that nodes which are close by never transmit simultaneously. CSMA-type MACs essentially create a guard zone around the receiver [12]. The traditional homogeneous PPP doesn't suit to this coexisting IEEE 802.15.4 networks, so we proposed PCMP model.

We consider WBSNs, each contains one receiver(CN: the cord node) and $\mathrm{N}$ transmitters(SN: sensor node). In our proposed model, the $\mathrm{CN}$ is placed at center of the human body. Some SN are deployed on a human body, forming a star topology with the CN such as Figure 1. Each SN has equal power and computation capabilities.

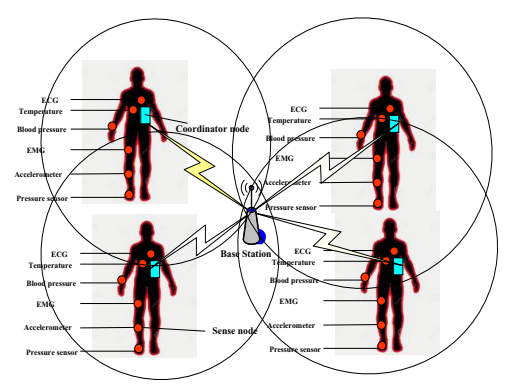

Fig. 1. System model.

The WBSN's locations are modeled as a stationary and isotropic Poisson cluster process (PCMP) coexisting in the $\mathrm{R}^{2}$ Euclidean space. The cluster process consists of the cluster centers (CNs) and cluster members ( $\left.\mathrm{SN}\right)$. The $\mathrm{CNs}$ spatial distribution model is a PPP with intensity $\lambda_{p p p}$ For the Poisson cluster process, each cluster member is uniformly distributed around the cluster center.

This PCMP is denoted by $\phi=\left\{\mathrm{x}_{i}, \mathrm{~m}_{i}, \mathrm{p}_{i}\right\}$, where

$\left\{\mathrm{x}_{i}\right\}$ denote the locations of the transmit points, is always assumed Poisson with positive and finite intensity $\lambda_{p p p}$.

$\left\{\mathrm{m}_{i}\right\}$ are marks, uniformly distributed on $[0,1]$.

$\left\{\mathrm{p}_{i}\right\}$ denote the transmit power by node $i$.

We denote target domain of area as M. Denoting by $\mathrm{K}$ the number of CNs in $\mathrm{M}$. $\mathrm{K}$ is Poisson distributed with mean $\lambda_{p p p} \mathrm{M}$ and probability mass function (pmf). $M$ is the standard Lebesgue measure of $\mathrm{M}$. So the numbers of $\mathrm{CN}$ in the domain $\mathrm{A}$ is:

$$
P(\mathrm{k}=\mathrm{n})=\frac{\left(\lambda_{p p p} M\right)^{k} e^{-\lambda_{p p p} M}}{n !}
$$

Define the contention domain:

$$
\mathrm{N}_{\mathrm{x}}=\left\{\left(\mathrm{x}_{i}, \mathrm{~m}_{i}, \mathrm{p}_{i}\right) \in \tilde{\phi}: \mathrm{p}_{i} \mathrm{~h}|| \mathrm{x}_{i}-\mathrm{x}_{j}||^{-\alpha} \geq \beta\right\}
$$

The medium access indicators $e_{i}=1\left(\Delta \mathrm{x}_{i} \in \mathrm{N}_{\mathrm{x}}, \mathrm{x}_{i}<\mathrm{x}_{j}\right)$

The lower the carrier-sensing threshold $\beta$ the higher the sensitivity of each transmitter to other transmissions occurring in the spatial domain, which increases the distances between simultaneously active transmitters and decreases the mutual interference at the expense of decreasing the spatial frequency reuse [13].

\section{Thinning method}


To consider a realistic medium-access control (MAC) layer algorithm, successful signal reception is based on the protocol model, and nodes employ carrier-sense multiple access [14]. One of the best choices for such scenarios is to use pure carrier sense multiple access (CSMA), which only incorporates channel sensing. In the CSMA protocol, every communication entity first senses the ongoing transmission in the channel and then determines when to start transmitting [15]. A node which wants to access the shared wireless medium senses its occupation and refrains from transmitting if the channel is already locally occupied.

Throughout this paper, we will say that the transmitter $x_{i}$ is older than the transmitter $x_{j}$ (or equivalently $x_{j}$ is younger than $x_{i}$ ) to denote that $m_{i}>m_{j}$. The hard core point process (HCPP) accounts only for the points having the lowest mark in their contention domains [16].

The classical HCPP leads to the following scenario: node $x_{1}$ is not retained because of it detects its neighbors $x_{2}$; moreover node $x_{2}$ in turn is not retained because it detects its neighbor $x_{3}\left(m_{1}>m_{2}>m_{3}\right)$, such as the nodes with marks $0.9,0.3,0.2$ in Figure 2. Consequently, in the Matern CSMA model neither $x_{1}$ nor $x_{2}$ is retained. But if $x_{3}$ is not the neighbor of $\mathrm{x}_{1}$, and if $x_{3}$ does not detect any of its neighbors then a more reasonable MAC would allow $x_{1}$ and $x_{3}$ to transmit simultaneously [17]. Such phenomena underestimate the density of the actual running network. Node is retained rules:

(1) $\left\{m_{i}<m_{j} \mid m_{i}, m_{j} \in \mathrm{B}_{x_{i}}(\beta)\right\}$

(2) $\left\{m_{j}<m_{d}<m_{l} \mid m_{d}, m_{l} \in \mathrm{B}_{x_{j}}(\beta) \backslash x_{j}\right\}$

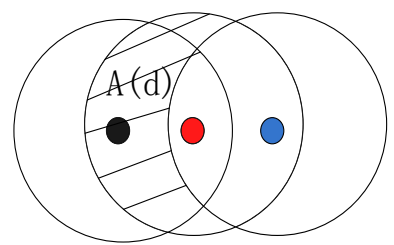
0.9
0.3
0.2

Fig. 2. The second neighbour node.

The shaded area:

$$
A(\mathrm{~d})=\pi \mathrm{R}^{2}-2 \mathrm{R}^{2} \arccos \left(\frac{d}{2 R}\right)+\frac{d}{2} \sqrt{4 d^{2}-\mathrm{R}^{2}}
$$

According to the distance properties of a PPP:

$$
\begin{aligned}
& 0<d<R \\
& E(\mathrm{~d})=\frac{1}{2 \sqrt{\lambda_{p p p}}} \\
& E(A(\mathrm{~d}))=\pi \mathrm{R}^{2}-2 \mathrm{R}^{2} \arccos \left(\frac{E(d)}{2 R}\right)+\frac{E(d)}{2} \sqrt{4 E(d)^{2}-\mathrm{R}^{2}}
\end{aligned}
$$

The probability of $x_{k}$ is the lowest mark in the $\mathrm{A}(\mathrm{d})$ :

$$
\mathrm{P}(\mathrm{A})=\mathrm{P}\left\{m_{k}<m_{u}, m_{u} \in\left(\mathrm{B}_{x_{j}}(\beta)-\mathrm{B}_{x_{i}}(\beta)\right) \backslash x_{j}\right\}
$$

The probability of the nodes retained: 


$$
\begin{aligned}
& p=\sum_{n=0}^{\infty} \frac{1}{n+1} \frac{\wedge_{B_{x_{i}}}^{n} e^{-\wedge_{B_{x_{i}}}}}{n !} \sum_{m=0}^{\infty} \frac{1}{m+1} \frac{\wedge_{A(\mathrm{~d})}^{m} e^{-\wedge_{A(\mathrm{~d})}}}{m !} \\
& =\frac{1-e^{-F}}{F} \frac{1-e^{-H}}{H} \\
& p_{P C M P}=\frac{1-e^{-F}}{F}\left(1+\frac{1-e^{-H}}{H}\right) \\
& \lambda_{P C M P}=\lambda_{P P P} p_{P C M P} \\
& =\lambda_{P P P} \frac{1-e^{-F}}{F}\left(1+\frac{1-e^{-H}}{H}\right)
\end{aligned}
$$

\section{Simulation results}

We have distributed many transmission nodes in the $20 \mathrm{~m}^{*} 20 \mathrm{~m}$ area according to certain density. Set the contain domain $R$ around the origin. If the closet node's distance is less than a certain value, the node with the smallest mark is retained.

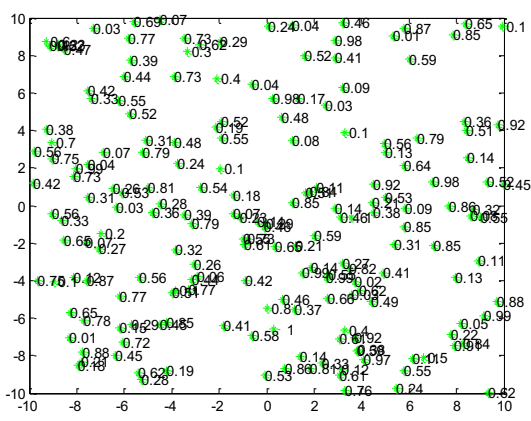

(a)PPP model.

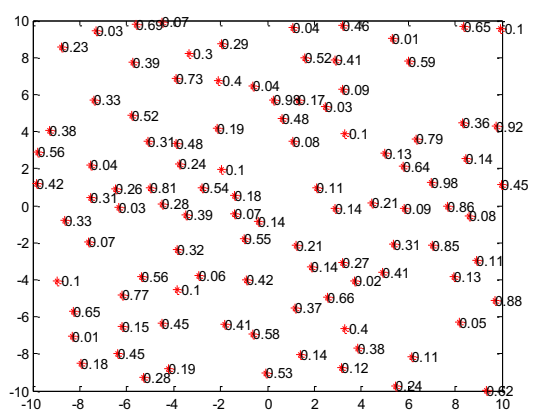

(b)PCMP model.

Fig. 3. PPP model and PCMP model.

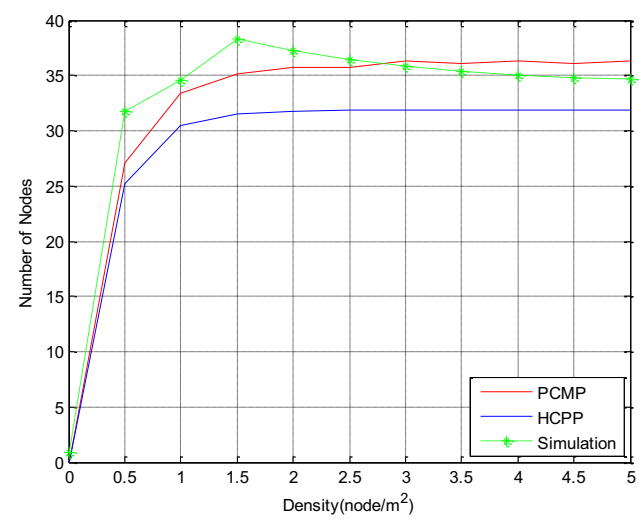

Fig. 4. The retained nodes of HCPP model and PCMP model.

Figure 3 compare the classical hard core point process and the PCMP via Monte Carlo simulations using Matlab to verify the accuracy of mode. We distributed many transmitter nodes in the 100 meters of area according to certain density. Set the contain domain is $2 \mathrm{~m}$. If the closet node's distance less than 2 , the node with the smallest mark is retained. The underestimation problem of HCPP model lead to the many of nodes is unselected, so in the Figure 4 the number of remaining nodes in PCMP model is much more than the HCPP mode. Their number of selected node is the same in the low density because the intersect probability of contain domain is very small. 


\section{Conclusion}

PCMP is derived by applying a specific thinning rule to a homogeneous Poisson point process in $\mathrm{R}^{2}$. In the following, the way of thinning a Poisson point process in order to obtain a moderate point process. The proposed model will be useful for capturing the intensity of simultaneously active nodes in a random CSMA wireless sensor networks.

APPENDIX A

$$
\begin{aligned}
& p=\sum_{n=0}^{\infty} \frac{1}{n+1} p\left(\left|\mathrm{~B}_{x_{i}}(\mathrm{R}) \backslash \mathrm{x}_{i}\right|=\mathrm{n}\right) \sum_{m=1}^{\infty} \frac{1}{m+1} p(|\mathrm{~A}(\mathrm{~d})|=\mathrm{m}) \\
& =\left(\frac{e^{-F}}{F} \sum_{n=0}^{\infty} \frac{F^{n+1}}{(\mathrm{n}+1) !}\right)\left(\frac{e^{-H}}{H} \sum_{m=0}^{\infty} \frac{H^{m+1}}{(m+1) !}\right) \\
& =\frac{1-e^{-F}}{F} \frac{1-e^{-H}}{H}
\end{aligned}
$$

Where $F=\wedge_{B_{x_{i}}}=\lambda_{p p p} \pi R^{2}$ is the mean number of nodes in $B_{x_{i}}, H=\wedge_{A(\mathrm{~d})}=\lambda_{p p p} E(\mathrm{~A}(\mathrm{~d})) \mathrm{E}(\mathrm{A}(\mathrm{d}))$ is the mean numbers of nodes in $E(\mathrm{~A}(\mathrm{~d}))$.

\section{References}

1. G.Z. Yang, Body Sensor Networks, Springer, (2006) .

2. A. Pantelopoulos, N.G. Bourbakis, A survey on wearable sensor-based systems for health monitoring and prognosis, IEEE Transaction on System, Man, and Cybernetics 40, (2010) .

3. M. Deylami, E. Jovanov, Performance Analysis of Coexisting IEEE 802.15.4-Based Health Monitoring WBANs, 34th Annual International Conference of the IEEE EMBS, (2012).

4. W. Lusheng, C. Goursaud, N. Nikaein,etc, Cooperative Scheduling for Coexisting Body Area Networks, IEEE Trans.Wireless Commun., (2012).

5. F. Martelli, Modeling Query-Based Wireless CSMA NetworksThrough Stochastic Geometry, IEEE Trans. Veh. Technol., 63, (2014).

6. B. Cho, K. Koufos, Bounding the Mean Interference in Matern Type II Hard-Core Wireless Networks, IEEE Wireless Commun. Letters, 2, (2013).

7. A. Busson, G. Chelius, J. Gorce, Interference Modeling in CSMA Multi-Hop Wireless Networks. (2009).

8. E. Hesham, H. Ekram, C. Sergio, Spectrum-efficient multi-channel design for coexisting IEEE 802.15.4 networks:a stochastic geometry approach. IEEE J. Sel. Areas Commun, 13,(2014).

9. F. Martelli, C. Buratti, R. Verdone, Modeling query-based wireless CSMA networks through stochastic geometry. IEEE Trans. Veh. Technol., 63,(2014).

10. F. Baccelli , B. Blaszczyszyn . Stochastic geometry and wireless networks, Volume II: applications. Paris, Now Press, (2005).

11. E. Hesham, H. Ekram, A Modified Hard Core Point Process for Analysis of Random CSMA Wireless Networks in General Fading Environments, IEEE Trans.Wireless Commun., 61, (2013).

12. S. Hamed, M. Pakravan, Analytical Modeling and Performance Analysis of Flooding in CSMA-Based Wireless Networks, IEEE Trans. Veh. Technol., 61, (2013).

13. S. Chiu, D. Stoyan, Stochastic geometry and its applications. Third Edition. UK, (2013).

14. P. Yiyang, L. Yingchang, K. Chan, Energy-Efficient Design of Sequential Channel Sensing in Cognitive Radio Networks: Optimal Sensing Strategy, Power Allocation, and Sensing Order, IEEE J. Sel. Areas Commun., 29, (2011).

15. J. Akhtman, L. Hanzo, Power versus bandwidth efficiency in wireless communications: the economic perspective. In:Proceeding of Proc. IEEE VTC-Fall09, (2009).

16. Y. Fengji, S. Yi, B. Sikdar, Scaling of Spatial Reuse and Saturation Throughput in a Class of MAC Protocols, IEEE Trans.Wireless Commun., 10, (2007).

17. W. Xiaofei, T. Berger, Spatial channel reuse in wireless sensor networks, Wireless Netw., 14, (2008) . 